journal club

\title{
2-Hydroxyglutarat-Magnetresonanzspektroskopie
}

\section{Neuer Marker für IDH-mutierte Gliome}

Hintergrund und Fragestellung: Für Gliome gilt die Kontrolle des klinischen Verlaufs neben der Magnetresonanztomografie (MRT) als Goldstandard für das Patientenmanagement. Andere physiologische, biochemische oder auch gewebebasierte Diagnostika wurden bis Dato nicht alltagstauglich entwickelt.

Mutationen des Gens für die Isozitratdehydrogenase 1 (IDH-1) führen dazu, dass Gliomzellen verstärkt 2-Hydroxyglutarat (2HG) produzieren. Das hat vielfältige diagnostische Bemühungen angestoßen, die IDH-Mutation bzw. 2-HG als Biomarker zu etablieren. In der hier diskutierten Studie wurde geprüft, ob die 2-HGMagnetresonanzspektroskopie (MRS) zur Diagnose und Verlaufskontrolle für Patienten mit IDH-mutierten Gliomen geeignet ist.

Patienten und Methodik: In der Studie von Changho Choi und

Originalie

Choi $\mathrm{C}$ et al. Prospective longitudinal analysis of 2-hydroxyglutarate magnetic resonance spectroscopy identifies broad clinical utility for the management of patients with IDH-mutant glioma. J Clin Oncol. 2016;34(33):4030-39. Kollegen wurde 2-HG mittels der MRS mit einer sehr hohen

untersucht. Weitere 38 Patienten wurden wiederholt während eines gemäß Bildgebung und Klinik stabilen Verlaufs untersucht, weitere 18 Patienten zum Zeitpunkt der Progression. Zusätzliche Patienten wurden unter und nach der Therapie analysiert.

Ergebnisse: In den MRS-Spektren fand sich ein erhöhtes Cholin im Vergleich zum Kreatin und eine Erniedrigung von N-Acetylaspartat sowie ein sehr kleiner 2-HG-Peak von variabler Höhe bei 2,25 PPM („parts per million“). Die Schwelle für eine zuverlässige Messung von 2-HG wurde mit 1 mmol bestimmt. Die 2-HG-Konzentrationen korrelierten mit dem Tumorgrad. Eine Unterscheidung zwischen IDH-mutierten Astrozytomen und oligodendroglialen Tumoren war nicht möglich. Die Volumenprogression wurde mittels 2-HG-MRS etwa sechs Monate früher vorhergesagt. Insbesondere bei oligodendroglialen Tumoren war ein Therapieansprechen auf die Chemotherapie mit Temozolomid oder Nitrosoharnstoffen mit einer Reduktion der 2-HG-Spiegel assoziiert. barkeit bei 138 Patienten während 511 einzelner Untersuchungen detektiert. 76 Patienten wurden seriell untersucht (median 5 Untersuchungen pro Patient; medianer Followup: 19 Monate). 60 Patienten wurden mit histologischer Sicherung und weitere 18 ohne
Schlussfolgerungen der Autoren: Die Studie zeigt die Möglichkeiten eines großflächigen Einsatzes der 2-HG-MRS auf. Bei technischer Reproduzierbarkeit und Nutzung sowohl in der regulären Patientenversorgung als auch in klinischen Studien scheint diese Technik ein vielversprechender diagnostischer, prognostischer und für das Patientenmanagement relevanter Parameter zu sein.

Schlüsselwörter: 2-HG-MRS - Gliom - IDH - Mutation

\section{- Kommentar von Wolfgang Wick, Heidelberg}

\section{"Ein erster Schritt in Richtung klinischer Routineanwendung"}

Die vorliegende, sehr systematische und umfangreiche Studie beantwortet eine große Zahl von Fragen, die für die 2-HGMRS relevant sind. Eine Schwelle für die Detektion von 2-HG mit den aktuell verfügbaren Techniken wurde definiert. Die Korrelation mit dem Tumorgrad ist aufgrund der Assoziation zur Zelldichte nachvollziehbar und erlaubt für zellreiche Neoplasien eine recht zuverlässige Bestimmung der IDH-Mutation auch ohne neurochirurgische Intervention. Formal erlaubt dies die sichere Diagnose eines IDH-mutierten Glioms ohne Biopsie. Für einige Patienten kann dieses Vorgehen Therapiekonzepte vor einer chirurgischen Intervention ermöglichen - oder zumindest in ausgewählten Fällen Optionen für eine gänzlich ohne neurochirurgische Intervention durchgeführte Therapie eröffnen. Ein systematischer Verzicht auf eine Gewebesicherung ist formal - wegen der weiteren molekularen Parameter und der prognostischen Relevanz von makroskopischen Resektionen - nicht empfehlenswert. Insbesondere bei einer Verbesserung der Therapiekonzepte für IDH-mutierte Gliome würde eine frühere Vorhersage eines Therapieversagens (Progression) durch die 2-HG-MRS allerdings vorzeitigere Therapiewechsel und damit prinzipiell bessere Verläufe ermöglichen.
In der Fachwelt ist diese Studie mit Respekt, aber auch mit Verwunderung aufgenommen worden. Viele renommierte internationale Einrichtungen erreichen derzeit nicht annähernd die für die in dieser Studie getroffenen Aussagen erforderliche Reproduzierbarkeit. Der Gruppe aus Texas kann dies sicherlich nicht zum Vorwurf gemacht werden. Eine breite, erfolgreiche Replikation dieser Daten ist wissenschaftlich und insbesondere mit Blick auf einen möglichen klinischen Einsatz aber notwendig.

Das Thema der Integration von metabolischen bzw. Biomarkerinformationen über elegante, nicht invasive Methoden wird durch diese Arbeit jedoch sicher stimuliert.

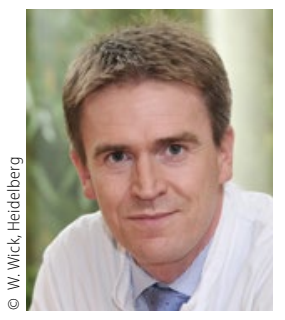

Prof. Dr. Wolfgang Wick

Ärztlicher Direktor, Neurologische Klinik, Universitätsklinikum Heidelberg wolfgang.wick@med.uni-heidelberg.de 